\title{
Evaluation of Immunoglobulin Sensing Function Using a Fullerene-Composite-Polymer-Coated Sensor Electrode
}

\author{
Takaaki Isoda*, Hikaru Sato ${ }^{1}$, Ikuko Urushibara ${ }^{1}$, Shigeru Uchida ${ }^{2}$, \\ Kouichi Kusuyama ${ }^{2}$, Tomoaki Kojima², Takeshi Asaka² and Ikumi Nitta² \\ Department of Life and Environment Engineering, Faculty of Environmental Engineering, \\ The University of Kitakyushu, 1-1 Hibikino, Wakamatsu, Kitakyushu 808-0135, Japan \\ ${ }^{1}$ AR'S Co. Ltd., 5-1 Yokohama Creation Square, \\ Sakaecho, Yokohama, Kanagawa 221-0052, Japan \\ ${ }^{2}$ ULVAC Coating Co. Ltd., 2804 Terao, Chichibu, Saitama 368-0056, Japan
}

(Received October 12, 2010; accepted December 27, 2010)

Key words sensor, fullerene, immunoglobulin, antigen, antibody

The detection of antigen-antibody reactions in normal saline solution measured using a chip-mounted source-drain electrode coated with a fullerene composite polymer layer containing an immobilized immunoglobulin $\mathrm{G}(\mathrm{IgG})$ molecule was investigated as a function of anti-IgG concentration. Five pairs of source-drain electrodes ( 5 wells) were fabricated on a glass substrate $(16 \mathrm{~mm} \times 40 \mathrm{~mm})$, in which the individual $\mathrm{Au} / \mathrm{Cr}$ $(1.0 / 0.1 \mu \mathrm{m}$ thickness) sensors had widths of $0.5 \mathrm{~mm}$ and an interelectrode interval of $1.5 \mathrm{~mm}$. The fabricated source-drain electrodes were further coated with an insulation layer comprising porous materials for use as an adsorbent for receiving IgG molecules. The sensor chip was equipped with a sensor signal analyzer that comprised either an amplifier circuit with a Miniship ${ }^{\mathrm{TM}}$ or a system in a packaged large-scale integration (LSI) device. Accuracy, as well as reproducibility for the detection of anti-IgG, was improved by the addition of fullerene to the insulation layer, which had a correlation coefficient for the calibration curve of $0.83-0.88$, whereas a fullerene-free insulation layer had a low correlation coefficient between $0.16-0.38$.

\section{Introctuction}

Various enzyme-linked immunosorbent assay (ELISA) methods for analyzing antigen and/or antibody molecules in a biological sample and for measuring the concentrations of target substances have recently been developed on the basis of specific antigen-

*Corresponding author: e-mail: isoda@env.kitakyu-u.ac.jp 
antibody reactions. As an example, the measurement of tumor necrosis factor-alpha (TNF- $\alpha$ ) levels using ELISAs has been reported to be useful in transplant research. ${ }^{(1)}$ Measurement of free serum human prostate specific antigen (PSA) in conjunction with total PSA, ${ }^{(2)}$ a quantitative assay for human leukocyte interferon with monoclonal antibodies, ${ }^{(3)}$ has also been carried out using ELISAs.

However, even though ELISA and fluorometric methods have very high detection levels (pg-ng/ml), they typically require a long time, from a few hours to a few days, to prepare primary and secondary antibodies and antigen reaction steps. In addition, commercially available ELISA kits and fluorescence microplate reader systems are expensive.

In the development of chemical sensors, many compact and low-cost sensors based on the measurement of conductivity changes have been proposed. The sensing of a number of chemical substances such as ethanol, ${ }^{(4)}$ urea, ${ }^{(5-7)}$ glucose, ${ }^{(8)}$ iron complexes, ${ }^{(9)}$ as well as hydrogen-ion exponent $(\mathrm{pH})^{(10)}$ and antigen-antibody reactions ${ }^{(11-15)}$ has been reported. These systems measure the concentration of a specific substance in solution, and it is possible to fabricate sensor-based chips using semiconductor technology, such as photolithography, and attach or integrate the sensors into an LSI. Particularly, ion-sensitive field-effect transistors (ISFETs) are widely employed as semiconductor sensors. Their construction is essentially sandwichlike, in which a silicon insulator layer is wedged between a source-drain electrode and a working or gate electrode. Furthermore, boron or phosphorus atoms are doped within the insulator layer to produce the semiconductor. The source-drain electrode current in ISFETs is changed by the field effect of the gate electrode originating from hydrogen ions.

We previously reported on the standard properties of a biosensor that had been mounted on a chip with a source-drain electrode, coated with an insulating layer of adsorbent particles for immobilizing antibody molecules. ${ }^{(16)}$ Figure 1(a) shows a schematic structure of a sensor-array chip. When a liquid is deposited on the surface of an insulator, an electric double layer occurs at the boundary surface; the insulator molecules become polarized, resulting in the formation of an opposing charge on the boundary surface between the liquid and the solid, and the reverse side of the insulator. Figure 1(b) shows the detection mechanism of an antibody and/or antigen molecule for sensor-array chips. In the case of a solution containing antigen molecules deposited on the surface of an insulator with immobilized antibodies, specific antigen and antibody reactions occur (Fig. 1(b)(A)) causing the charge at the reverse side of the insulator to increase (Fig. 1(b)(B)). The relationship between the source-drain electrode current and the sensor voltage follows Ohm's law. On the basis of this principle, a sourcedrain electrode produces a signal in response to changes in current originating from the antigen-antibody reactions, such as rat anti-IgE and $\operatorname{IgE}$, leukotriene B4 and antileukotriene B4, and biotin maleimide and avidin, at the boundary surface of the insulator layer. ${ }^{(16)}$ In the next stage of sensor development, the sensor-array chip with immobilized antibodies proposed previously will be applied to measure the concentrations of immunoglobulins. Accuracy, as well as repeatability for sensor measurement, will be improved if a state of polarization of all sensor arrays can be controlled equally by adding an insulating layer on the electrode. 
(a)

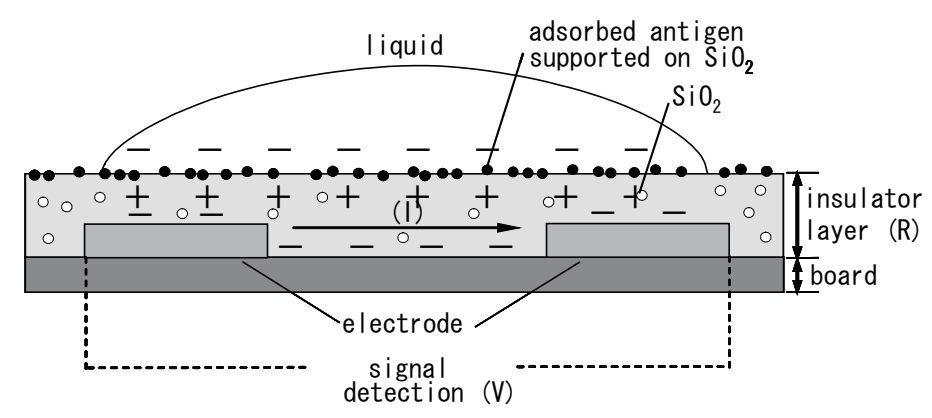

(b)

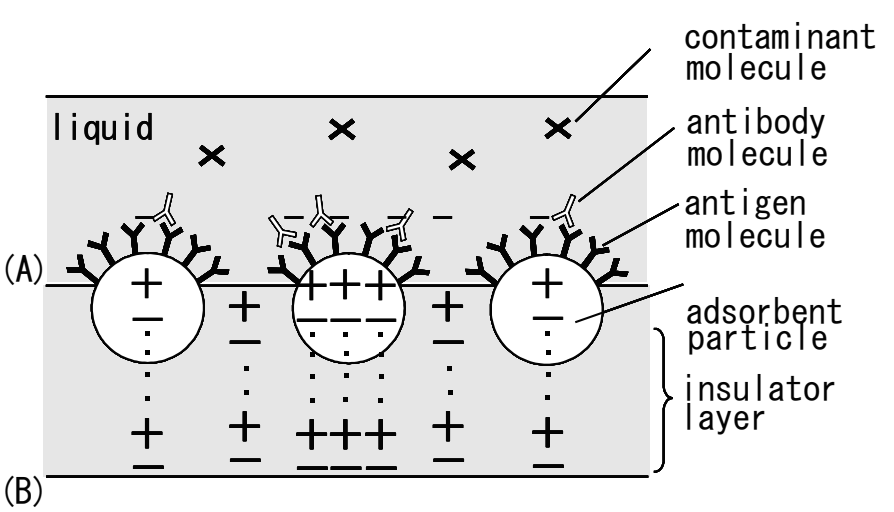

Fig. 1. Schematic structure of a sensor-array chip (a) and detection mechanism of an antibody molecule (b).

In this study, the detection of antigen-antibody reactions in a normal saline solution using a chip-mounted source-drain electrode coated with a polymer layer and immobilized IgG molecules was investigated as a function of anti-IgG concentration. In particular, repeatability of the detection of anti-IgG by the addition of fullerene into a polymer insulation layer was examined. The sensing functions were determined by measuring the source-drain electrode voltage when the surface of the insulator was in contact with a selection of phosphate-buffered saline (PBS) and/or anti-IgG solutions at various concentrations. On the basis of the above results, the detection ability and advantages of a fullerene-composite-polymer-coated sensor electrode are discussed.

\section{M aterials and M ethods}

\subsection{Fabrication of sensor electrode}

Figure 2 shows the fabrication steps of a sensor-array chip involving the preparation of source-drain electrodes coated with an insulation layer. $\mathrm{A} \mathrm{Cr}$ adhesion layer $(0.1$ $\mu \mathrm{m}$ thick) and $\mathrm{Au}$ layer $(1 \mu \mathrm{m}$ thick) were coated successively onto a glass substrate 


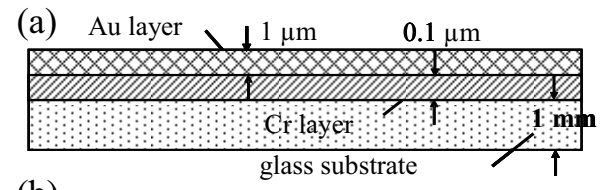

(b)
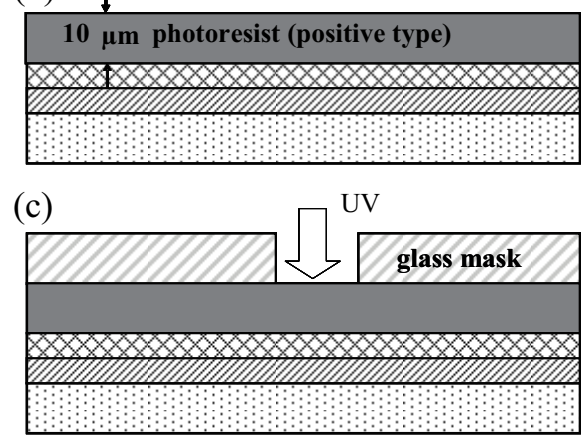

(d)

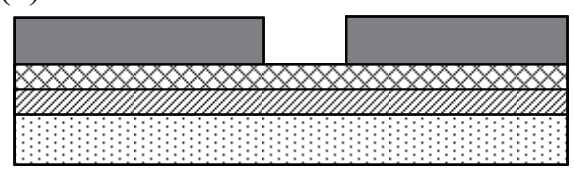

(e)
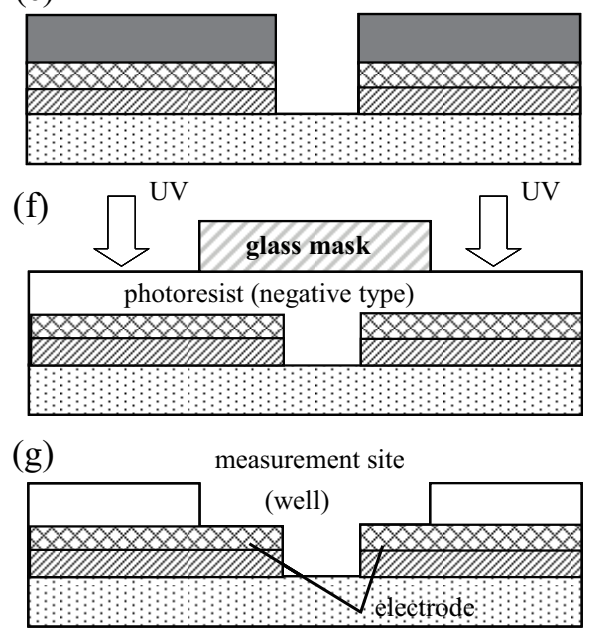

(h) insulator layer adsorbent

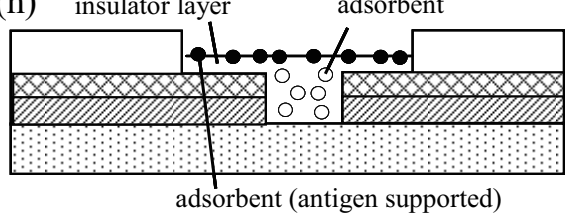

Fig. 2. Fabrication steps of a sensor-array chip.

(16 $\mathrm{mm} \times 40 \mathrm{~mm}, 1 \mathrm{~mm}$ thick) by sputtering (Fig. 2(a)). A positive-type photoresist was first spin-coated on the Au surface (Fig. 2(b)), and then patterned by irradiating the photoresist through a Cr-coated soda-lime glass mask with ultraviolet (UV) light (Fig. 2(c)). Following irradiation, the exposed regions of the resist were removed using a $25 \%$ tetramethylammonium hydroxide (TMAH) solution (Fig. 2(d)). The underlying Au regions were then chemically etched using a $\mathrm{KI}^{-} \mathrm{I}_{2}$ solution $\left(\mathrm{KI} / \mathrm{I}_{2}=1 / 1 \mathrm{~g}\right.$, $17 \mathrm{wt} \%$; Fig. 2(e)). Patterning of the $\mathrm{Cr}$ layer was carried out using a ceric ammonium nitrate solution [5\% (w/v)] for chemical etching (Fig. 2(e)). The remaining photoresist layer was removed by washing extensively in acetone. A negative-type photoresist was spin-coated on the micropatterned surface again, and then patterned by irradiating the photoresist through a second Cr-coated glass mask with UV light (Fig. 2(f)). After irradiation, the unexposed regions of the resist were removed using a developer, and a microwell structure on each electrode was formed (Fig. 2(g)).

An insulator layer was formed on the source-drain electrode by first spin-coating a polymer dissolved in a solvent (hereafter referred to as the binder resin) with $\mathrm{SiO}_{2}$ particles or adsorbent $\left(10 \mu \mathrm{m} \phi\right.$, specific surface area: $\left.200 \mathrm{~m}^{2} / \mathrm{g}\right)$ on the electrode, and then dried at room temperature for $5 \mathrm{~min}$ (Fig. 2(h)). Details of the preparation method are described in the next section. Mouse IgG $(100 \mu \mathrm{g} / \mathrm{ml}$, Sigma $)$ dissolved in PBS 
was then deposited at $5 \mu \mathrm{l}$ on each well of the sensor array (Fig. 2(h)). After adsorption treatment for $5 \mathrm{~min}$, the sensor-immobilized antibodies were then washed with distilled water.

Figure 3 shows a photograph of the fabricated sensor-array chip (Fig. 3(a)) and the mask layout (Fig.3(b)). Five wells of $2.9 \mathrm{~mm} \phi$ were arranged on both sides of a chip over a $16 \mathrm{~mm} \times 40 \mathrm{~mm}$ area. A well unit was composed of source (positive terminal) and drain (negative terminal) electrodes arranged within an area of $2.9 \mathrm{~mm} \phi$. The length, width, and interelectrode spacing of each sensor electrode were fixed at 0.7, 0.5, and 1.5 $\mathrm{mm}$, respectively. The sensor electrodes were connected by pads arranged at the edges of the chip, which were in turn connected to an amplification circuit in the sensor signal analyzer.

\subsection{Preparation of a fullerene composite polymer}

Figure 4 shows a schematic of fullerene doping into a polymer material for the purpose of electrode coating. It has been reported that fullerene soot can be partially dissolved in o-dichlorobenzene, toluene, and benzene at 25, 2.9, and 1.5 $\mathrm{g} / \mathrm{L}$, respectively.(17) In contrast, it was difficult to dissolve fullerene in polar solvents such as carbon tetrachloride, ${ }^{(18)}$ dichloromethane, ${ }^{(18)}$ dichlorohexane, ${ }^{(18)}$ acetone, ${ }^{(18)}$ and isopropanol. ${ }^{(19)}$ Figure 4(a) shows a fullerene solubilized component in a solvent (C60S) used as an additive in binder resin. In this case, C60S is a uniform composite in a binder resin layer, and a thin layer forms a homogeneous phase. In Fig. 4(b), C60S is dissolved in a poor and polar solvent, and used as an additive in the binder resin. In this case, aggregated fine particles originating from $\mathrm{C60S}$ are dispersed in the binder resin layer, and a thin layer forms a heterogeneous phase.

Figure 5 shows a preparation scheme for fullerene doping into polymer material. Commercially available fullerene (Nanom purple: $98 \% \mathrm{C}_{60}$; Frontier Carbon Co.) was used and two types of insulation layer, a C60S uniformity dissolution layer or a C60S fine-particle-doped layer, were fabricated on the sensor electrode.
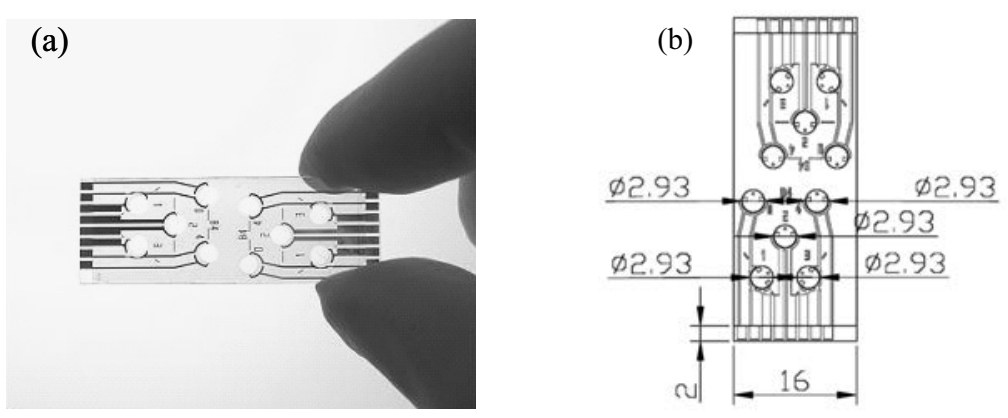

Fig. 3. Photograph of a sensor-array chip. (a) Production model "Well sensor Type II" (ULVAC Coating Co.) and (b) the mask layout. 
(a) Case1

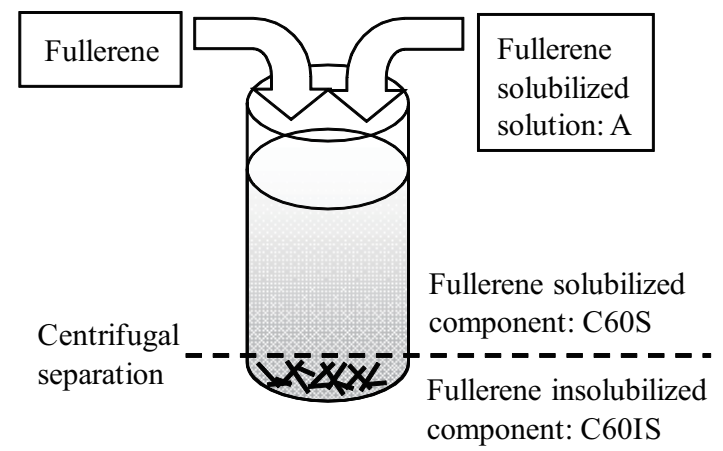

(b) Case2

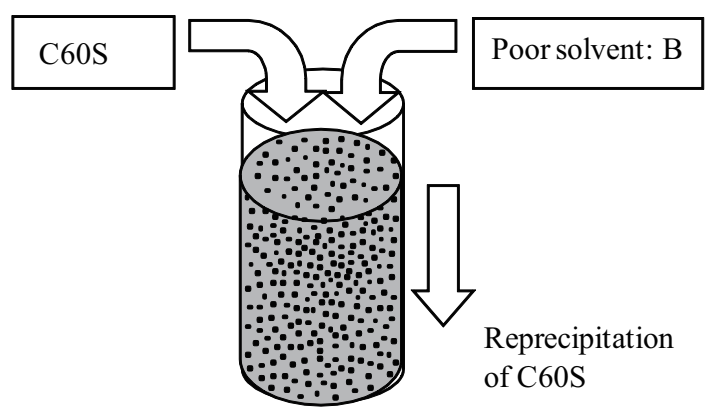

Fig. 4. The schematic of fullerene doping into polymer material for the purpose of an electrode coating. Case 1 is a use of fullerene solubilized component in a solvent (C60S) (a) and case 2 is a use of fullerene reprecipitation component made by C60S with poor solvent.

\subsection{Evaluation of sensor property using PBS}

Three concentrations of PBS were prepared $(0.2 \times, 1.8 \times$, and $5.0 \times)$. A $4 \mu$ volume of $0.2 \times$ PBS was deposited on five wells of a chip and the PC software for the wireless sensor-screening system ${ }^{(16)}$ (Biosensor System 2006 Model $^{\mathrm{TM}}$, AR'S Co.) was started. The average sensor voltage $\left(\mathrm{E}_{0.2}(\mathrm{~N})(\mathrm{N}\right.$; well Nos. 1-5)) from each sensor between the measurement time interval of $0-30 \mathrm{~s}$ was obtained.

A $4 \mu \mathrm{l}$ volume of $1.8 \times$ PBS was continuously deposited on each well for $120 \mathrm{~s}$. The concentration of the droplet on each well was diluted in $1 \times$ PBS (major composition: $\left.\mathrm{NaCl} 1.38 \times 10^{-1} \mathrm{moll}^{-1}\right)$. The average sensor voltage $\left(\mathrm{E}_{1.0}(\mathrm{~N})(\mathrm{N}\right.$; well Nos. 1-5)) from each sensor between the measurement time interval of $90-120 \mathrm{~s}$ was obtained.

A $4 \mu$ volume of $5 \times$ PBS was deposited on each well for $120 \mathrm{~s}$ and the concentration of the droplet on each well was diluted such that the final concentration of PBS was $2.3 \times$. The average sensor voltage $\left(\mathrm{E}_{2.3}(\mathrm{~N})(\mathrm{N}\right.$; well Nos. 1-5)) from each sensor at $90-120 \mathrm{~s}$ was obtained. 


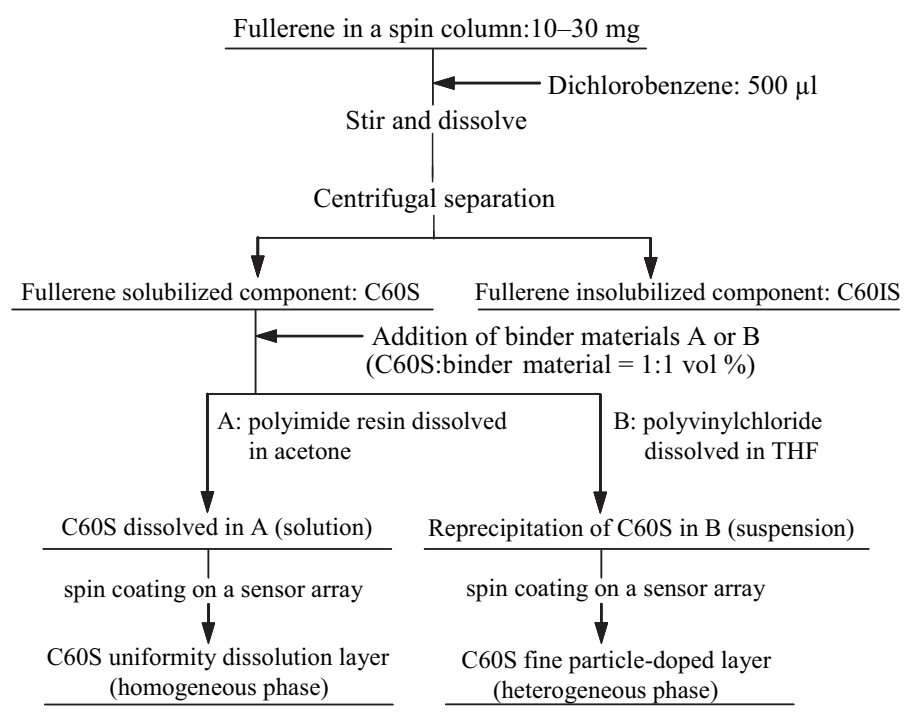

Fig. 5. Preparation of fullerene doping into polymer material.

The sensor voltages from each of the five wells, $E_{0.2}(\mathrm{~N}), \mathrm{E}_{1.0}(\mathrm{~N})$, and $\mathrm{E}_{2.3}(\mathrm{~N})$, were further averaged and the detection voltages at each PBS concentration, $\mathrm{E}_{0.2}, \mathrm{E}_{1.0}$, and $\mathrm{E}_{2.3}$, were obtained.

The average detection values, $\mathrm{E}_{0.2}, \mathrm{E}_{1.0}$, and $\mathrm{E}_{2.3}$, were plotted against each PBS concentration. Detection precision was evaluated using a coefficient of correlation value by the linear least-squares method. When the sensor ability, as well as detection precision, was higher, the correlation coefficient approached 1.0.

\subsection{Evaluation of a sensor property using anti-IgG}

As explained in the previous section, mouse IgG was immobilized on each sensor surface before sensor evaluation. Mouse anti-IgG (Dako Co.) was dissolved in 5× PBS at the five given concentrations and prepared as test samples.

A $4 \mu \mathrm{l}$ volume of $0.2 \times$ PBS was deposited in five wells on a chip, and the PC software for the wireless sensor-screening system was started. The measurement time was $120 \mathrm{~s}$ and the average sensor voltage from each sensor between 0-120 s was obtained. Five concentrations of each test sample were deposited in each well (12 $\mu$ l per sample) over $240 \mathrm{~s}$. The value of the relative response, $R$, was evaluated using eq. (1):

$$
R=\left(E_{2}-E_{0}\right) /\left(E_{1}-E_{0}\right) \text {. }
$$

In this instance, $E_{0}$ was the average sensor voltage from each sensor between 0-120 s with $0.2 \times$ PBS. $E_{1}$ was the maximum sensor voltage from each sensor when measuring the test sample. $E_{2}$ was the average sensor voltage from each sensor between 120 $240 \mathrm{~s}$ when measuring the test sample. The $\mathrm{R}$ value was plotted against each anti-IgG 
concentration. Detection precision was evaluated using the correlation coefficient by the linear least-squares method.

\section{Results}

\subsection{Fullerene composite polymer as a coating material for a sensor electrode}

Figure 6 shows an optical microscopy image of the interelectrode spacing of the fullerene-composite-polymer-coated electrode. Figure 6(a) shows a C60S uniformity dissolution doping layer in accordance with the preparation scheme in Fig. 5(A). Figure 6(b) shows C60S with a C60IS mixture layer, as a reference sample in Fig. 6(a). The C60S composite can be dissolved in a binder-polymer solution and a uniformity layer forms (Fig. 6(a)). It is clear that the C60IS composite cannot be dissolved in a binderpolymer solution and a nonuniform layer is formed (Fig. 6(b)). Figure 6(c) shows a C60S fine-particle-doped layer in accordance with the preparation scheme in Fig. 5B. Figure 6(d) is a phase-contrast image derived from Fig. 6(c). The black dot marked with arrows in Fig. 6(c) and the black dots in Fig. 6(d) are agglomerated C60S particles. These particles are not crystalline and the particle size was approximately $10 \mu \mathrm{m}$.
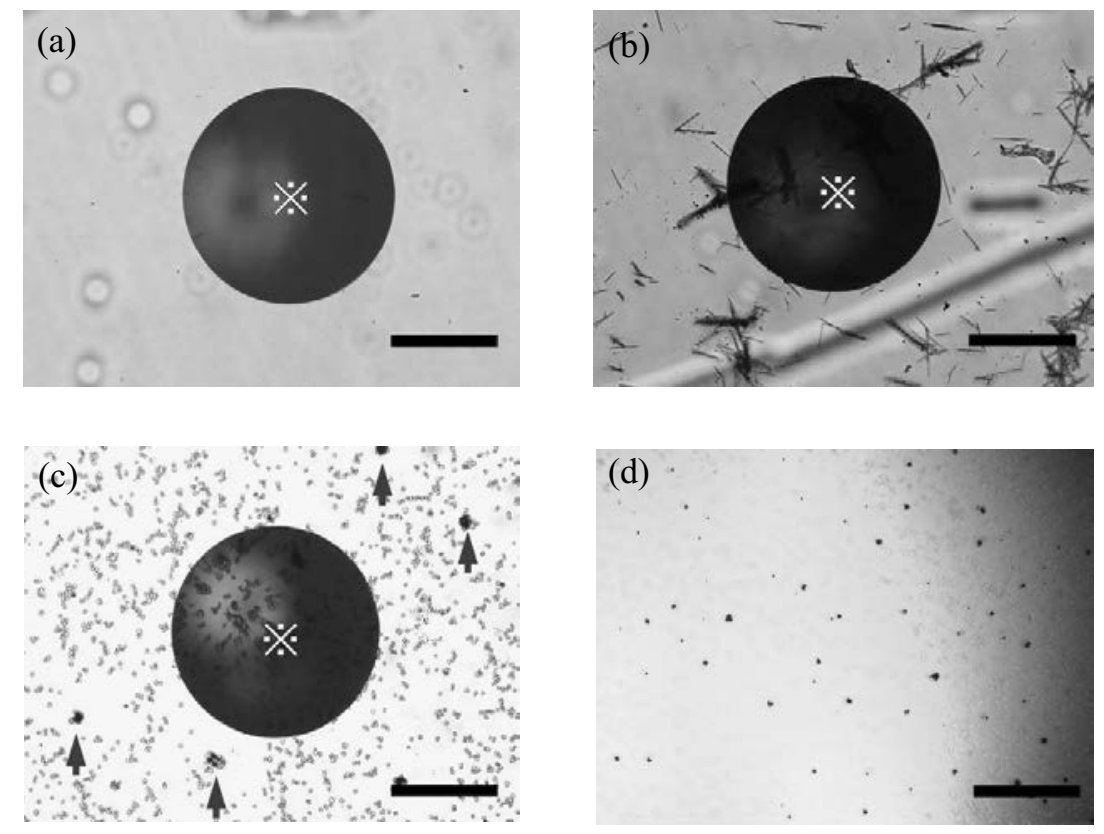

Fig. 6. Microscopy images of C60S-doped polymer-coated electrode. C60S uniformity dissolution doping layer (a), C60S+C60IS-doped layer (b), and C60S fine- particle-doped layer (c and d). (d) is a phase image. Scale bar for each image indicates $150 \mu \mathrm{m}$. The center dot in (a)(c) indicates the Au surface. The black dots marked with arrows in (c) and the black dots in (d) are agglomerated particles from C60S. 
3.2 Effect of the fullerene composite in the binder resin for sensor electrode coating

Figure 7 shows the effects of the amount of fullerene added to the C60S component for coating of the sensor electrode versus sensor voltage. The label "C60S" indicates a sensor electrode coated with the C60S component and a polyimide binder resin in accordance with the preparation scheme in Fig. 5(A); an example of this is shown in Fig. $6(a)$. The label "C60S+C60IS (reference)" indicates a sensor electrode coated with $\mathrm{C} 60 \mathrm{~S}$ and $\mathrm{C} 60 \mathrm{IS}$ components in a polyimide binder resin and an example of this is shown in Fig. $6(b)$.

Figure 7(a) shows the results from distilled water (DW) as measured using two types of sensor. For DW measurement, the detection voltage was low regardless of C60S and/or C60S+C601S doping in the binder resin, with values of $700 \mathrm{mV}$ for the C60Scoated electrode and 700-800 mV for the C60S+C60lS-coated electrode. The dielectric polarization was small at the interface of an insulator layer in contact with DW, the socalled nonaqueous electrolyte. ${ }^{(20)}$

Figure 7 (b) shows the results when $1 \times$ PBS was measured using two types of sensor. When the binder-resin-coated-sensor electrode, as well as the $\mathrm{C} 60 \mathrm{~S}$ nondoped insulation layer, was evaluated, the detection voltage increased to 1,350-1,550 $\mathrm{mV}$ when the binder-resin-coated sensor electrode was evaluated. In contrast, $10 \mathrm{mg}$ of C60 for the preparation of the $\mathrm{C} 60 \mathrm{~S}$ and/or $\mathrm{C} 60 \mathrm{~S}+\mathrm{C} 60 \mathrm{IS}$ components markedly increased the sensor voltage to $1,850 \mathrm{mV}$ for the $\mathrm{C} 60 \mathrm{~S}$-coated electrode and $1,700 \mathrm{mV}$ for the $\mathrm{C} 60 \mathrm{~S}+\mathrm{C} 60 \mathrm{~S}$ coated electrode. This result indicates that the dielectric polarization at the interface of an insulator layer in contact with an aqueous electrolyte is markedly enhanced by the addition of $\mathrm{C} 60 \mathrm{~S}$ and/or $\mathrm{C} 60 \mathrm{~S}+\mathrm{C} 60 \mathrm{IS}$ to the binder resin. However, the detection voltages decreased with increasing amounts of $\mathrm{C} 60 \mathrm{~S}$ and/or $\mathrm{C} 60 \mathrm{~S}+\mathrm{C} 60 \mathrm{IS}$ components in the insulating layer. At the present stage, the reason is not clear. However, the changes
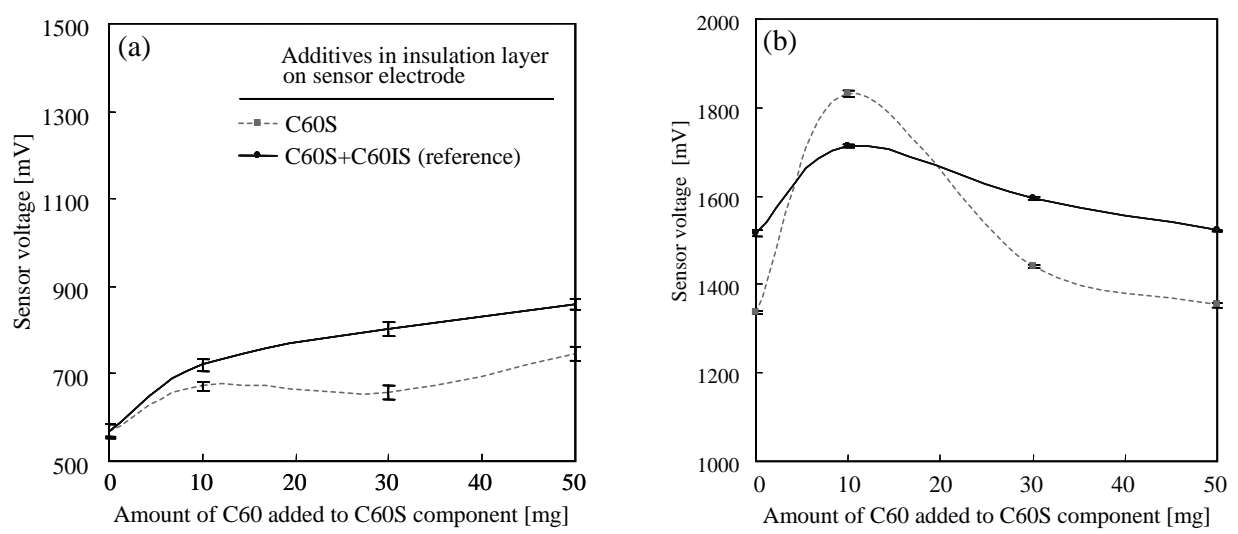

Fig. 7. A mount of fullerene added to the $\mathrm{C} 60 \mathrm{~S}$ component of the coating material for the sensor electrode vs. sensor voltage. M easurement samples were water (a) and $1 \times$ PBS (b). 
in the detection voltage using the C60S-coated electrode were larger than those using the $\mathrm{C} 60 \mathrm{~S}+\mathrm{C} 60 \mathrm{IS}$-coated electrode. It is estimated that the difference in the aggregation of the fullerene affected the polarization state of the insulation layer.

3.3 Effect of additives on the insulation layer of the sensor electrode for detection on sodium ions

Figure 8 shows the relationship between the detection voltage from a sensorarray chip and PBS concentration. The compositions of the insulator layer on the sensor electrode included a polyimide binder resin (Fig. 8(a), nondoped layer), C60S dissolved in a binder resin (Fig. 8(b), C60S-doped layer), and C60S+C60IS in a binder resin (Fig. 8(C), C60S+C60IS-doped layer). Each sensor showed a strong correlation between the detection voltage and PBS concentration, having correlation coefficients of 0.9959 for the nondoped layer, 0.9960 for the C60S-doped layer, and 0.9996 for the C60S+C6015-doped layer. A major component of 1 XPBS is the sodium ion. Therefore, the concentrations of the sodium ions as well as the PBS concentration show a linear
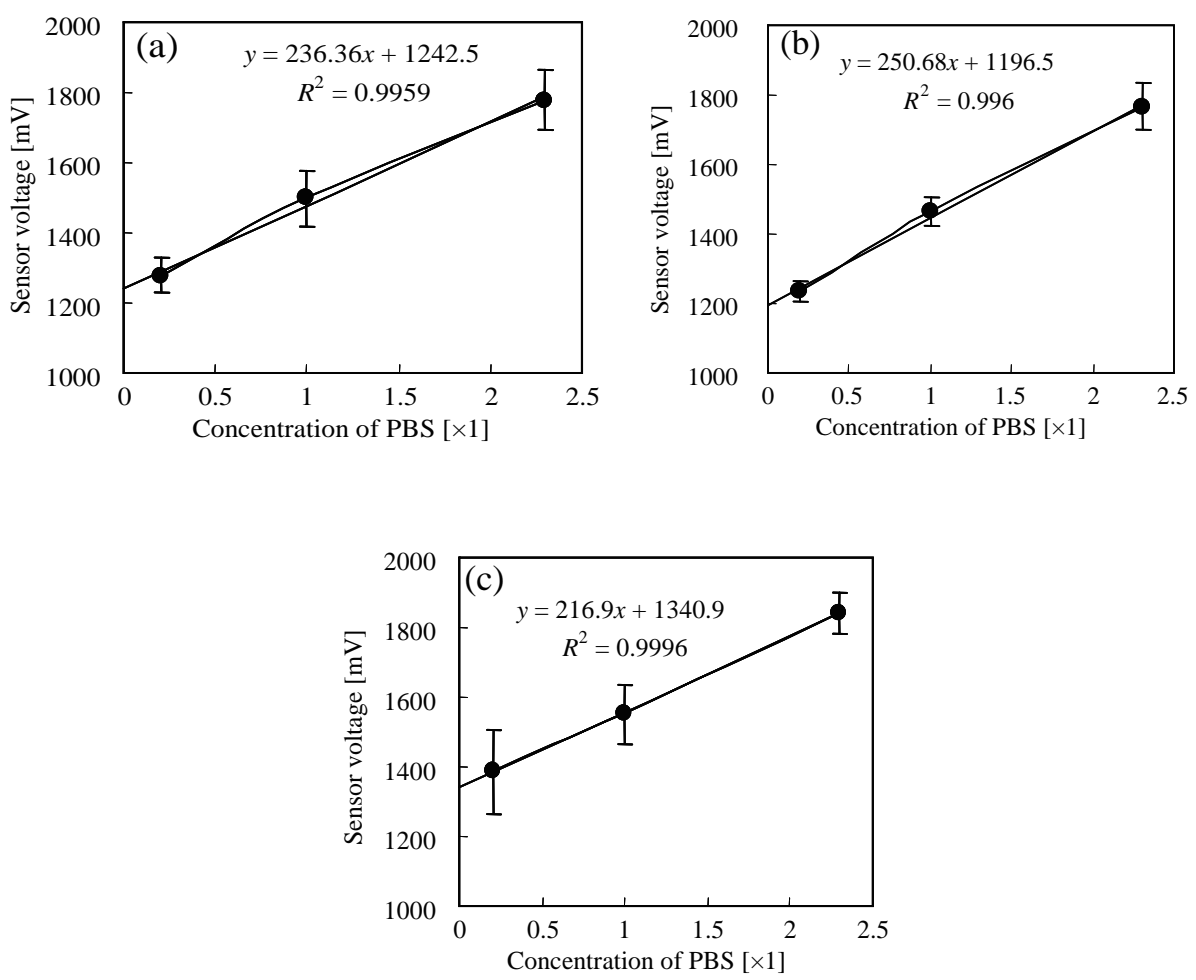

Fig. 8. Relationship between detection voltage from a sensor-array chip and concentration of PBS: (a) polyimide (binder composition), (b) C60S dissolved in a binder composition, and (c) $\mathrm{C} 60 \mathrm{~S}+\mathrm{C} 60 \mathrm{IS}$ in a binder composition (reference). 
response to dielectric polarization at the interface of the insulator layer. However, it was noted that the margin of error in each evaluation was different. This result indicates that the addition of the $\mathrm{C} 60 \mathrm{~S}$ component in the binder resin reduces the detection error.

3.4 Effect of additives on the insulating layer of the sensor electrode on detection of antigen

Figure 9 shows the relationship between detection voltage from a sensor-array chip and mouse anti-IgG concentration. The compositions of the insulator layer on the sensor electrode included a polyvinylchloride binder resin (Figs. 9(a) and 9(b), nondoped layer) and C60S fine particles in a binder resin (Figs. 9(c) and 9(d), C60S-doped layer). In this case, the C60S-doped layer was prepared in accordance with the preparation scheme shown in Fig. 5, and an example of this can be seen in Figs. 6(c) and $6(d)$. Evaluation of the sensor experiment was carried out twice on one chip to confirm reappearance.
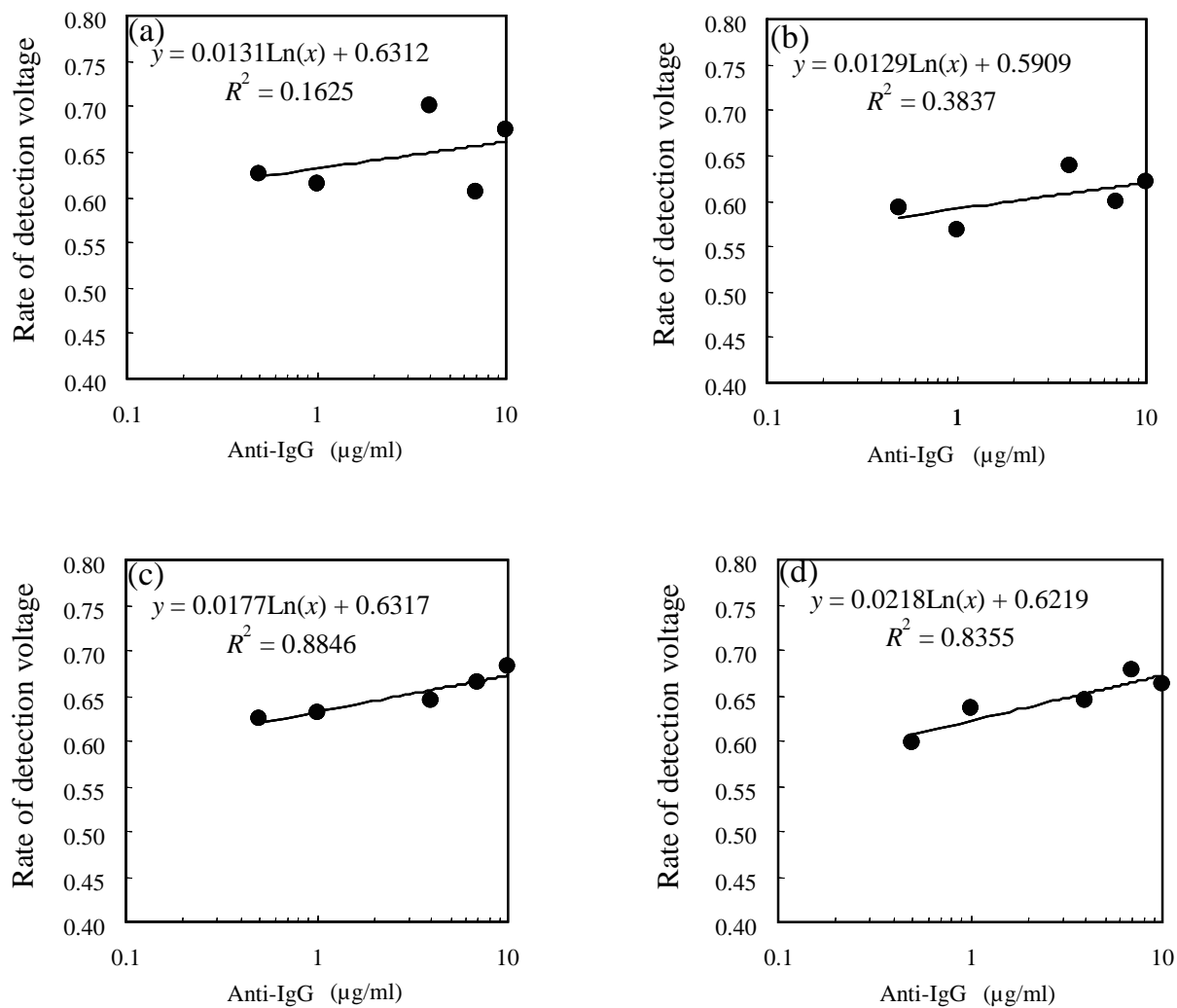

Fig. 9. Relationship between detection voltage from a sensor-array chip and concentration of anti-IgG in PBS. M aterial of the sensor surface layer: polyvinylchloride $(a, b), C 605$ fine-particledoped layer in a binder composition (c, d). 
For the nondoped layer, the correlation between the detection voltage and anti-IgG concentration was low, with a coefficient of 0.1625 for the left side of the chip and 0.3837 for the right side of the chip. In contrast, the correlation between detection voltage and anti-IgG concentration was improved using the C60S-doped layer, with correlation coefficients of 0.8846 on the left side and 0.8355 on the right side of the chip. This result indicates that the addition of C60S fine particles to the binder resin also decreases the error rates for the detection of antigen-antibody reactions.

\section{Disassion}

The sensor described in this study does not require a gate electrode, and only a polymer-based insulator layer with a simple structure is necessary. ${ }^{(16)}$ In our previous paper, we reported that when a liquid is deposited on the surface of an insulator, an electric double layer arises at the boundary surface. Here, the insulator molecules become polarized, resulting in the formation of opposing charges on the boundary surface between the liquid and solid, and on the reverse side of the insulator. Therefore, a source-drain electrode generates a response as a result of changes in both current and charge. ${ }^{(16)}$ In the case of antibodies immobilized on adsorbent particles on the insulator surface, the detection of antigens can be explained on the basis of the previously mentioned polarization mechanism. ${ }^{(20)}$ In accordance with the above mechanism, the voltage of a source-drain electrode coated with an insulator layer comprising the C60S and/or C60S fine particle components was found to affect the stabilization of the charge on the surface of the sensor electrode.

A chip-mounted microelectrode sensor coated with an insulator layer dissolved in fullerene was investigated with respect to its properties relating to the detection of antibody/antigen interactions. This study proved useful in evaluating the developed sensor-array system as a packaged LSI M iniship and as a sensor-screening system for the detection of signals from a sensor-array chip. When a sample solution containing antigen molecules was deposited onto a sensor with immobilized antibodies, the converted digital signals from the sensors were transmitted to a receiver located $5 \mathrm{~m}$ away. The concentrations of sodium ions and PBS demonstrate a linear response using sensor array chips regardless of the insulation layer on the electrode. However, the margin of error was decreased when the C60S component in the binder resin was used as an additive.

The correlation between detection voltage and anti-IgG concentration was higher using the C60S-doped layer than using the nondoped layer. In the case of a solution containing antigen molecules deposited on the surface of an insulator with immobilized antibodies, specific antigen and antibody reactions occurred, causing the charge at the reverse side of the insulator to increase. The relationship between the source-drain electrode current and the sensor voltage followed $0 \mathrm{hm}$ 's law. In line with this principle, a source-drain electrode produces a signal in response to changes in current originating from the antigen-antibody reactions at the boundary surface of the insulator layer. In accordance with the above mechanism, the voltage of a source-drain electrode coated with an insulator layer comprising the C60S and/or C60S fine particle components was found to affect the stabilization of the charge on the boundary surface of the polymer layer on a sensor electrode. 


\section{Acknowledgment}

This work was supported by funds from the Japan Science and Technology Corporation via the Research Seeds Quest Program.

\section{R eferences}

1 C. P. M aury and A. M. Teppo: J. Exp. M ed. 166 (1987) 1132.

2 R. Junker, B. B randt, C. Zechel and G. A ssmann: Clin. Chem. 43 (1997) 1588.

3 T. Staehelin, C. Stähli, D. S. H obbs and S. Pestka: M ethods in Enzymology 79 (1981) 589.

4 Y. G. Lee and T. C. Chou: Biosens. Bioelectron. 20 (2004) 33.

5 U. Bilitewski, W. Drewes and R. D. Schmid: Sens. A ctuators, B 7 (1992) 321.

6 A. Steinschaden, D. A damovic, G. J obst, R. G latz and G. U rban: Sens. A ctuators, B 44 (1997) 365.

7 N. F. Sheppard J r. D. J. M ears and A. Guiseppi-Elie: Biosens. Bioelectron. 11 (1996) 967.

8 G. E. De Benedetto, F. Palmisano and P. G. Zambonin: Biosens. Bioel ectron. 11 (1996) 1001.

9 A. V. Legin, E. A. Bychkov and Y. G. V lasov: Sens. Actuators, B 15 (1993) 184.

10 N. F. J. Sheppard, M . J. Lesho, P. M cN ally and A. S. Francomacaro: Sens. A ctuators, B 28 (1995) 95.

11 Method and device for detecting analyte electrically and quantitatively, Japanese Patent 2005-37376.

12 Electrode sensor for measuring immunity of an antigen body, J apanese Patent 2002-333419.

13 Electrode sensor for measuring immunity of an antigen body, J apanese Patent 2002-174163.

14 Electrode sensor for measuring immunity of an antigen body, J apanese Patent 2003-262601.

15 Electrode sensor for measuring immunity of an antigen body, J apanese Patent 2002-174614.

16 T. Isoda, I. U rushibara, M. Sato, H. U emura, H. Sato and N. Y amauchi: Sens. A ctuators, B 129 (2008) 958.

17 A. S. W alter and M. T. James: J. Chem. Soc. Chem. Commun. 15 (1993) 1207.

18 R. S. Ruoff, D. S. Tse, R. M al hotra and D. C. Lorents: J. Phys. Chem. 97 (1993) 3379.

19 D. Heymann: Carbon 34 (1996) 627.

20 T. I soda, H. M akimoto, H. Imanaga, R. Imamura, J. Pawlat and T. U eda: Sens. A ctuators, B $123(2007) 805$. 